\title{
„Everything was very neat": Abject Art zwischen Kunstgeschichte und Politik
}

\section{Anja Zimmermann}

Eingegangen: 25. Mai 2021 / Angenommen: 20. September 2021 / Online publiziert: 25. Oktober 2021

(C) Der/die Autor(en) 2021

Zusammenfassung Der Beitrag beleuchtet die Rezeption von Abject Art in der Kunstgeschichte und darüber hinaus. Als Kategorie, die die Kunstwissenschaft und -kritik ab den 1980er-Jahren den Schriften der französischen Psychoanalytikerin Julia Kristeva entnahm, diente sie der Kennzeichnung von Kunstwerken, die u.a. mit Repräsentationen des Körpers arbeiteten, die klassischen Idealen widersprachen. Verweise auf Verletzungen, Traumata, aber auch Körperflüssigkeiten und -grenzen waren Themen dieser Arbeiten oder wurden an ihrem Beispiel aus ästhetischer Sicht kritisch analysiert. Besonderes Augenmerk legt der Text auf politische Reaktionen auf Kunstwerke der Abject Art, die als „obszön“, ,abstoßend“ oder ,ekelhaft“ beschrieben und damit abgewehrt werden. Der Beitrag verdeutlicht an konkreten Beispielen, vornehmlich aus den USA, wie die künstlerischen Strategien, etwa die Verwendung bestimmter Werktitel, eine Rezeption der Werke als abjekt in Gang setzen und wie umgekehrt aber auch Werke, die bei ihrer Entstehung nicht in Bezug zu abjekten Themen gesetzt wurden, später als abjekt gedeutet wurden.

Schlüsselwörter Abject Art · Kunst und Politik · Julia Kristeva · Culture Wars · Körperbilder in der Kunst 


\title{
“Everything was very neat": abject art, art history, and politics
}

\begin{abstract}
The article concentrates on the reception of Abject Art in art history and beyond. As a category which art history and art criticism took from the writings of the French psychoanalyst Julia Kristeva from the 1980s onwards, it served to identify works of art that for example worked with representations of the body that contradicted classical ideals. References to injuries, trauma, but also body fluids and body boundaries were topics of these works or were critically analyzed using their example from an aesthetic point of view. The text pays special attention to political reactions to works of abject art that are considered "obscene", "repulsive" or "disgusting" and are thus rejected. The article uses concrete examples, mostly in the US, to illustrate how artistic strategies, such as the use of certain work titles, initiate a reception of the works as abject and how, conversely, also art works that were not related to abject topics when they were created, later would be interpreted as abject.
\end{abstract}

Keywords Abject art - Art and politics · Julia Kristeva $\cdot$ Culture wars · Body images in art

\section{Abject Art zwischen Kunstkritik und Politik}

Das Abjekt begann seine kunstgeschichtliche Karriere im Laufe der 1980er-Jahre. Im Rekurs auf Julia Kristevas Pouvoirs de l'horreur (1980) wurden Ausstellungen wie Abject Art im New Yorker Whitney Museum (1993) oder L'Informe: Mode d'Emploi (1996) im Pariser Centre Pompidou entwickelt. Letztere setzte sich zwar ausdrücklich von Kristeva ab, wurde aber als konkurrierendes Theoriemodell ebenfalls in den Kontext der Debatten um Abjektion und Kunst diskutiert. Die Kunst, die in diesen und anderen Ausstellungen gezeigt wurde, rückte Themen in den Fokus, die mit „niederen“, mitunter ekelerregenden Materialien assoziiert waren oder zeigte Visualisierungen des Körpers als verletzt, tot, abstoßend. Werke wie Cindy Shermans großformatige Fotografien verschimmelter, an Erbrochenes erinnernder Substanzen zählten ebenso dazu wie Werke der Bildhauerin Kiki Smith, deren Skulpturen kriechende, scheinbar kotbeschmierte Körper zeigten.

In der Rezeption ließ sich jedoch schon kurz nach Beginn der kunstwissenschaftlichen Anwendung des Begriffs der Abjektion auf diese Arbeiten eine Skepsis ausmachen. Sie besagte, dass die Kunst, die durch ihre Themen, die verwendeten Materialien oder auch die formale Gestaltung beanspruchte, den Ekel in die ästhetische Erfahrung zu überführen, genau an diesem zentralen Moment ihrer eignen Produktionslogik scheitere, denn ,wirklicher“ Ekel entstehe in der Begegnung mit den Werken der Abject Art nicht. So betonte z.B. Winfried Menninghaus, Werken der Abject Art gelinge es nicht, zu jener Ekelzone vorzudringen, die die ,klassische Grundlegung der Ästhetik in Ekel-Tabus und in der schönen Oberfläche eines ekellosen Idealkörpers“ immer verleugnet habe (Menninghaus 2002). Dabei griff er eine Überlegung des Kunsthistorikers Denis Hollier auf, der beim Besuch der New Yorker Abject-Art-Ausstellung bemerkt hatte: „When I saw the ,Abject Art“ show 
at the Whitney, I thought: What is abject about it? Everything was very neat; the objects were clearly art works. They live on the side of the victor." (Menninghaus 2002, S. 176). Er beschrieb damit die Erfahrung mancher Museumsbesucherin, den white cube der Ausstellungsmoderne sauber, wohlgenährt, gesund durchstreifen zu können und dabei auf Werke zu treffen, die zwar die erwähnten Ekeltopoi repräsentieren, selbst aber rezipiert werden können, ohne sie tatsächlich auch als Angriff auf die eigene körperliche Ekelgrenze zu erleben. Die Kritik zielte daher darauf, dass die Gestaltung der infrage stehenden Arbeiten - trotz allen Ekelbezugs - ebendiesen Ekel bei ihren Rezipient*innen gerade nicht zulasse, sei es durch makellose, glatte Farboberflächen wie in den „Ekel“"-Fotografien Cindy Shermans oder schlicht durch die sublimierende Wirkung weißer, glatter, makelloser Ausstellungsräume, in denen die Begegnung mit den Werken stattfindet.

Die von Hollier und Menninghaus formulierte skeptische Beobachtung gegenüber dem Subversionspotenzial des Abjekten in der Kunst ist daher sicherlich angebracht. Aber eben nur einerseits. Denn andererseits sind die erwähnten und weitere Arbeiten der Abject Art durchaus in der Lage, heftige Reaktionen auszulösen. Während es also zweifellos eine Rezeptionsweise abjekter Kunst gibt, bei der das Ekelpotenzial der Arbeiten ins Leere läuft, ist dies in anderen Rezeptionskontexten nicht so. Legendär in diesem Zusammenhang ist ein Auftritt des republikanischen Abgeordneten D'Amato, der vor dem amerikanischen Kongress gegen Andres Serranos großformatige Fotoarbeit Piss Christ protestierte und dabei einen Katalog des Künstlers zerriss. Immer wieder sind solche und vergleichbare (Re-)Aktionen, die jenseits des engen Zirkels kunsthistorischer Rezeption angesiedelt sind, als irrelevant bei der Frage nach dem Ekel in der Kunst abgetan worden. Dies liegt teils daran, dass sie in erster Linie als moralische Urteile verstanden wurden, d.h. als Rezeptionserfahrungen, die den Ekelbegriff nur vorschützen, um damit eine besonders heftige Verachtung der abgewehrten Kunstwerke auszudrücken. Eine nähere Beschäftigung mit dieser „kunstfernen“, scheinbar allein im politischen Raum angesiedelten Rezeption im Rahmen einer ästhetisch argumentierenden Auseinandersetzung mit Abject Art lohne daher nicht. ${ }^{1}$

Wie hier jedoch argumentiert werden soll, ist eine solche Diagnose schon allein deswegen fragwürdig, weil sie eine außerkünstlerische, politische Sphäre außer Acht lässt, die als Zielpunkt vieler Arbeiten ernst genommen werden sollte. Verzichtet man darauf, wird die Ausdeutung der Arbeiten im „rein“ Ästhetischen gleichsam selbst einem „Reinigungsverfahren“ unterzogen. Die aus kunsthistorischer Sicht wenig qualifizierten Reaktionen von Personen, die nicht als Kunstexpert*innen, sondern z.B. als konservativ-fundamentalistische Politiker*innen auf die Kunst reagieren, gehören in dieser Sichtweise jedenfalls nicht dazu, belegen aber gerade das politische Aktivierungspotenzial von Abject Art. Daher - und dies soll im Folgenden an Rezeptionsbeispielen, bei denen die erwähnten ,,außerkünstlerischen“ Ekelbekundungen den Ausgangspunkt der Analyse bilden oder explizit in diese einbezogen werden, gezeigt werden - sollten diese Reaktionen nicht vorschnell aus einer kunsthistorischen Auseinandersetzung mit Abject Art aussortiert werden. Vielmehr sind

\footnotetext{
${ }^{1}$ So kommt dieser politische Strang der Rezeption in vielen Artikeln zur Abject Art als eigentliches Thema kaum vor, so z. B. auch nicht bei Menninghaus (2002).
} 
Abb. 1 Andres Serrano: Piss Christ. Fotografie, 1987 (C) Paula Cooper Gallery). Aus: https://prometheus.unikoeln.de/de/image/genfc51c61fbe3d54e7204ce0b3f4 $8 \mathrm{a} 53836338559 \mathrm{~b} 8$

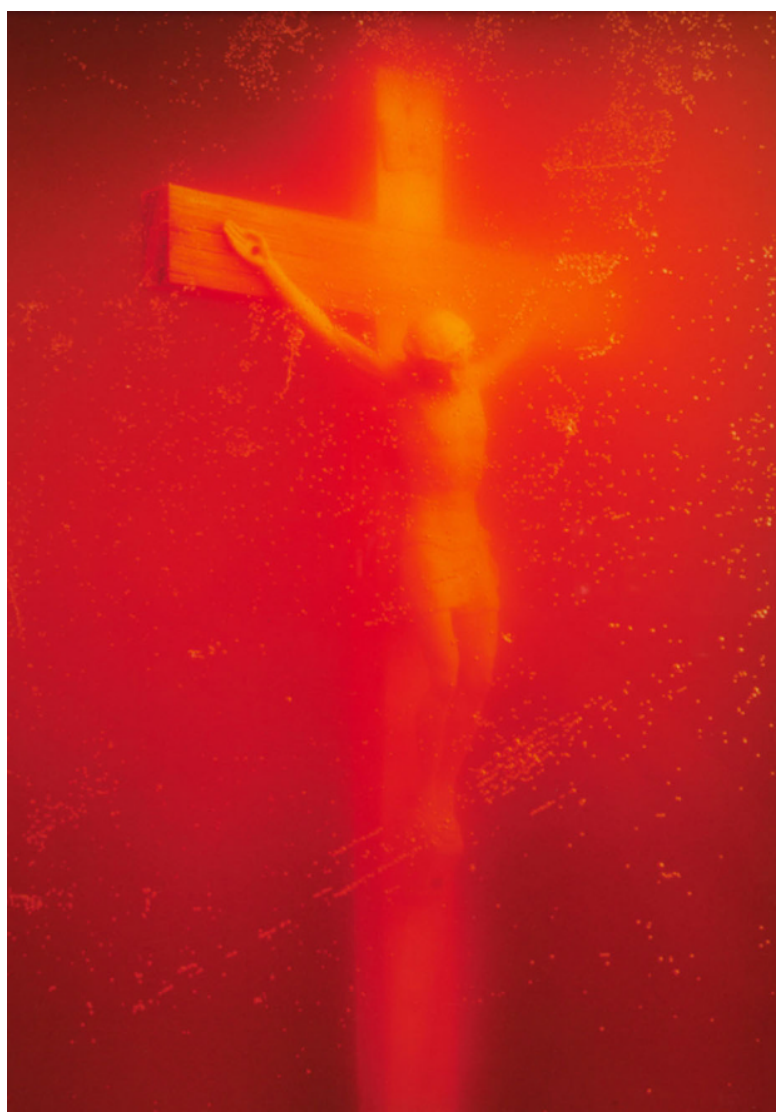

sie, wie ebenfalls gezeigt werden soll, integraler Teil dessen, was die Kunstgeschichte als Abject Art beschreibt und ausstellt.

\subsection{Piss Christ: Religion, Kunst, Staat}

Eine langjährige Klassikerin der Abject Art und ihrer politischen Debatten ist die bereits adressierte Fotografie des US-amerikanischen Künstlers Andres Serrano aus dem Jahr 1987 (Abb. 1). Das großformatige Werk zeigt ein Kruzifix schwebend in einer schimmernden Flüssigkeit, mutmaßlich im Urin des Künstlers. Unter dem Titel Piss Christ wurde die Arbeit zunächst in Los Angeles ausgestellt, um dann in Richmond, Virginia die Aufmerksamkeit einer christlichen Organisation, der American Family Association, zu erregen, die mit entsprechenden Protesten dagegen begann (Zimmermann 2001). Die Proteste weiteten sich aus und gipfelten schließlich in dem ebenfalls bereits benannten ikonoklastischen Auftritt des Republikaners Alphonse D'Amato. Andere Politiker agierten in vergleichbarer Weise. Besonders erfolgreich war dabei der konservative Senator Jesse Helms, dessen Aktivitäten schließlich zum sogenannten Helms Amendment führten, das die staatliche Förderung „obszöner“ 
Kunst untersagte. Immer wieder kam es zu vergleichbaren Reaktionen, etwa 1997, als Piss Christ auf dem Arts Festival in Melbourne in Australien gezeigt wurde. Hier war es eine religiös-fundamentalistische Gruppe namens Victorian Parliamentary Christian Fellowship, die im Rekurs auf die früheren Proteste in den USA Vergleichbares forderte (Young 2000).

Eine Reihe von Art-World-Rezipient*innen wies bei der Beschäftigung mit Piss Christ dagegen auf formale Fragen der Bildgestaltung wie Komposition, Farbe etc. hin. Hervorgehoben wurde etwa, dass die Farben ,,possibilities of pleasure, quite apart from prejudice against the contemplation of ,piss “" ermöglichen würden (Cumming 1998). In der Tat setzt die Fotografie durch die goldglänzende, strahlende Qualität der Umgebung des scheinbar schwebenden Kruzifixes stark auf den ästhetischen Genuss. Freilich ist damit eine in der Ästhetik des Ekels lang tradierte Überlegung aktiviert, die besagt: ,some artworks [...] are capable of incorporating the arousal of disgust into an experience of beauty, and that they do so without sacrificing the visceral power of the apprehension." (Korsmeyer 2011, S. 160). Es waren mutmaßlich u.a. diese anziehenden Oberflächeneffekte der Fotografie, die Betrachter*innen wie Denis Hollier dazu brachten, die eigentlich mit der Körperflüssigkeit Urin assoziierten Ekelgefühle nicht wahrzunehmen und sich vielmehr stets der Tatsache bewusst zu bleiben, dass die Begegnung mit dem Kunstwerk einen nicht mit der Substanz selbst, also dem Urin, sondern lediglich mit dessen Repräsentation konfrontiert. Auch bei anderen Arbeiten aus dem Kontext der Abject Art lokalisiert sich das, was als abjekt qualifiziert, fast nie auf Materialebene (d.h. in der verwendeten Substanz), sondern in Hinblick auf das „Motiv“. Bei Piss Christ kommt noch etwas Weiteres hinzu. Es ist allein der Titel, der eine Assoziation mit dem Abjekt nahelegt. Ohne Titel wäre die Arbeit, so ist anzunehmen, niemals auf dem Radar der fundamentalistischen Sittenwächter, die sie immer wieder zum Ziel ihrer Kampagnen machten, aufgetaucht. Die blasphemische Zielrichtung des Werks ist überdeutlich gemacht, allerdings eben nicht auf Werkebene, sondern ausschließlich durch die Betitelung. Inwiefern dieser und seine Verweigerung (,ohne Titel“) in der Kunst der Moderne untrennbar von der „Bedeutung“ des Werks ist, seine Rezeption entscheidend zu strukturieren mag und zugleich der strategischen Positionierung des Künstlers oder der Künstlerin dienen kann, ist bekannt (Bann 1985; Bruch 2005; Vogt 2006). Hier kommt dem Titel eine Beglaubigungsfunktion zu: Was wir sehen ,ist“" Urin. Kritiker wie Hollier, die der Abject Art absprechen, tatsächlich Ekelgefühle auslösen zu können, tun dies auf Basis eines Titels, der in diesem Fall anzeigt, dass eine nach den Regeln der Abjektion (Kristeva 1982) mit Ekel assoziierte Substanz „da“ sei. Aber auch beim Anblick von Piss Christ von heftigen Ekelgefühlen geschüttelten Politiker wie Joseph D'Amato oder Jesse Helms, reagieren, wie sie berichten, auf das Begriffsamalgam „Piss“ und „Christ“ und kaum auf das Bild „selbst“, das sie in diesem Fall vermutlich ebenfalls als „,very neat“ hätten beschreiben können. Die Arbeit des Künstlers stellt mithilfe des Titels, wie in der Moderne und Gegenwartskunst üblich, hier nicht nur selbst eine deutende Rahmung bereit, sondern hebt den Zusammenhang von ästhetischer und außerästhetischer Thematisierung des Ekels hervor. Nicht selten wird hier von Text und Kontext gesprochen: Das Werk ist in diesem Fall der ästhetische „Text“, die Reaktionen auf dieses werden dem außerästhetischen „Kontext“" zugeordnet (Culler 
1988). Allerdings gibt es gute Gründe anzunehmen, dass im Fall von Abject Art diese Trennung nur bedingt hilfreich ist. Denn Piss Christ scheint seine abjekte Wirkung fast ausschließlich bei jenen entfaltet zu haben, bei denen es aufgrund des Titels auf eine blasphemische Wirkung rechnen konnte. Damit situiert sich die Arbeit ganz bewusst innerhalb der seit den 1980er-Jahren in den USA schwelenden Culture Wars (Hartman 2019). „Ekel“ erweist sich damit - und in dieser Hinsicht weicht die Arbeit von der Behauptung einer anthropologisch konstanten Ekel-Erfahrung ab - als trotz allem zutiefst kulturell verankerte Größe, zu der auch die kunstgeschichtliche Praxis des Ausstellens, Zeigens und Deutens gehört. Die heftigen Reaktionen, die eine Arbeit wie Piss Christ außerhalb einer kunsthistorischen Rezeption im eigentlichen Sinne auslöste, gehören daher untrennbar sowohl zum Werk als auch zu dessen Potenzial zur Ekel-Evokation.

\title{
1.2 Abject Art als Rahmung: Deutungsstrategien
}

Abject Art ist, ebenso wie alle anderen stilistisch oder inhaltlichen Klassifizierungen der Kunstgeschichte, Ergebnis einer Zuschreibungspraxis. Anders als Kristevas

\begin{abstract}
Abb. 2 Eva Hesse: Unititeld or not yet, Netztaschen, transparentes Papier, Metallgewichte, Schnur, 1966. Aus: A. Zimmermann (2001). Skandalöse Bilder - Skandalöse Körper: Abject Art vom Surrealismus bis zu den Culture Wars. Berlin: Reimer, S. 50
\end{abstract}

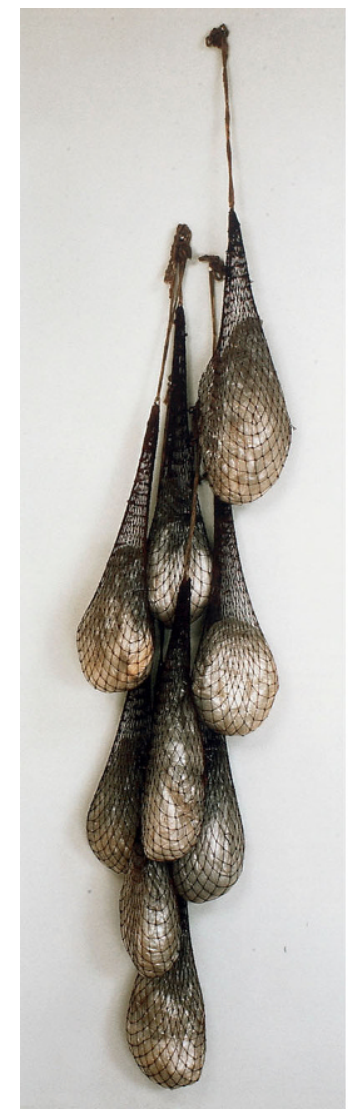


immer wieder zitiertes Beispiel für das Abjekt, die Haut, die sich auf der langsam erkaltenden Milch bildet, handelt es sich bei Kunstwerken, auch wenn diese z.B. die erwähnte Milchhaut zum Thema machen, um etwas kategorial Anderes. Sie lassen sich nicht bruchlos mit der „Wirklichkeit“ verrechnen, sondern liefern einen Überschuss, etwa an Distanzierungsmöglichkeiten, die sie von dieser unterscheiden. Dies bedeutet aber auch, dass das, was die Kunstgeschichte und ihre Institutionen als Abject Art, etwa durch Ausstellungen, präsentieren, maßgeblich erst durch diese dazu gemacht werden (Zimmermann 2001). Dies ist für den hier verfolgten Zusammenhang auch deswegen wichtig, weil sich hieran zeigen lässt, dass die Verhandlung des Abjekten in der Kunst immer auch eine Verhandlung darüber ist, was eine Gesellschaft als ,,abjekt“ diskutiert, und was die Kunstgeschichte selbst in ihren Kanon einschließt oder verwirft. Insofern kann es keine abjekte Kunst geben, die sich im Hinblick auf eine überhistorische Kategorie des körperlichen Ekels bestimmen ließe. Die oben skizzierten unterschiedlichen Ekelreaktionen auf Serranos Piss Christ im Kunstkontext und in den politischen Auseinandersetzungen offenbaren dies. Zu belegen ist es aber auch durch die hier bereits mehrfach erwähnte Ausstellung mit dem Titel Abject Art des New Yorker Whitney Museums. Die Kunstwerke, die die Kurator*innen dieser Ausstellung für ihr Projekt zusammengetragen hatten, waren zuvor nicht selten in ganz anderen Zusammenhängen gesehen worden, wobei eine Verknüpfung mit Ekelthematiken keineswegs von vornherein immer gegeben war. So wurden beispielsweise die skulpturalen Arbeiten der Bildhauerin Eva Hesse (Abb. 2) in den 1960er-Jahren „Chain Polymers [genannt, AZ] - not ,modular mess‘ or ,messy neatness“ or , pathetic objects ‘... no, not ,abject objects “ either" (Wagner 1994, S. 261), wie die amerikanische Kunsthistorikerin Anne Wagner betont. Anfang der 1990er-Jahre allerdings wurden ebenjene Arbeiten dieser Künstlerin, die die strengen Geometrien der Minimal Art durch biegsame und elastische Materialien aufzulösen begannen, als ,abjekt“ etikettiert. Wie Wagners Bemerkung zeigt, lässt sich eine solche Lesart, die in den Arbeiten vor allem eine (weibliche) Gegenposition zur (männlichen) minimalistischen Cleanness erkennen wollte, deutungsgeschichtlich datieren und ergibt sich daher nicht ,,aus dem Werk selbst“. Es wäre allerdings falsch, daraus zu schließen, dass die Nichtbeachtung der abjekten Qualitäten von Hesses Werk durch die zeitgenössische Rezeption besser oder plausibler wäre. Vielmehr macht Wagners Beobachtung letztlich klar, dass die Bezeichnung Abject Art mehr Deutung als Beschreibung ist. Welche kunsthistorischen Strategien mit einer solchen Deutung verbunden sein können, darum soll es im folgenden letzten Abschnitt gehen. Zur Verdeutlichung der Rolle, die Rahmung, Präsentation und Labeling spielen, sei hier noch auf einen Zwischenfall verwiesen, der erneut die Seite der Rezeption mit ins Spiel bringt, die mit dem Verweis auf die „Ekelhaftigkeit“ einzelner Kunstwerke, deren Präsentation und Förderung beschneiden oder verhindern will. Das Beispiel ist auch deswegen instruktiv, weil es zugleich erhellt, wie flüchtig die Zuschreibungen des Transgressiven an die Kunst im Wechsel politischer Zeitläufte sind. So wurden in den 1960er- und 1970er-Jahren mehrere Arbeiten des US-amerikanischen Künstlers Ed Kienholz als obszön beanstandet. Politiker beschrieben sie anlässlich einer Ausstellung im Los Angeles County Museum of Art im Jahr 1966 als ,repugnant“ (Silk 1997, S. 275; Zimmermann 2001, S. 180-185). In der Folge, nach dem Öffentlichwerden des Konflikts, erreichten 
das Museum eine Reihe an Beschwerden von Besucher*innen. Allerdings stellte sich heraus, dass diese auf einer Verwechslung beruhten. Die Beschwerden bezogen sich auf Kunstwerke des 17. und 18. Jahrhunderts, die nackte Körper zeigten. Diese Kritik konnte allerdings nur noch Spott auf sich ziehen und entfaltete keine kunstpolitische Wirkung, belegte sie doch, dass diejenigen, die sie äußerten, die Kunst des 20. Jh. nicht von der des 17. Jh. unterscheiden konnten. Die Kritik blieb folgenlos, weil diese älteren Kunstwerke den Prozess der „Kunstwerdung“ inklusiver musealer Institutionalisierung erfolgreich durchlaufen hatten, sodass selbst eine nachträgliche Kennzeichnung als ,abstoßend“ wirkungslos bleiben musste. Kienholz' Arbeit Back Seat Dodge '34 (1964), der Anlass für die damalige Kritik, wurde übrigens 1997 ohne jede vergleichbare Reaktion in New York ausgestellt - im Whitney Museum, in dem 1993 die Abject-Art-Ausstellung zu heftiger politischer Kritik geführt hatte.

\subsection{Körperbilder: Der weibliche Körper als abjekt}

Wie aber kam es dazu, dass eine Künstlerin wie Eva Hesse, deren Arbeiten vermutlich für diejenigen, die mit Abject Art nicht vertraut sind, wenig ,Ekelhaftes“ an sich haben, in einer Ausstellung mit diesem Titel auftauchte? Die Beantwortung dieser Frage führt zu einem weiteren Aspekt der Rezeption des Abjekts in Kunstgeschichte und -theorie. Bereits bei Kristeva (1982) war die Theorie des Abjekts auch eine, die Geschlechterdifferenz mit reflektierte. Betrachtet man die Rolle, die Abject Art in der kunsthistorischen Theorielandschaft spielte und spielt, dann lässt sich zusammenfassend feststellen, dass es über die Aktivierung dieses Begriffs in vielen Fällen gelang, die Kategorie Geschlecht dort zum Thema zu machen, wo sie zuvor keine oder nur eine implizite oder problematische Rolle gespielt hatte. Vor allem gelang es durch den Verweis auf das Abjekt, selbst wiederum auf Ungleichzeitigkeiten und Ungleichheiten in der Rezeption aufmerksam zu machen. Diese lassen sich auf drei Ebenen ausmachen:

Erstens scheint der Umgang mit abjekten Themen und Materialien, z. B. (eigenen) Körperflüssigkeiten wie sie etwa auch für Serrano kennzeichnend war, in der Rezeption bei Künstlerinnen tendenziell anders eingeordnet zu werden als bei ihren männlichen Kollegen. Während für männliche Künstler die Arbeit mit dem Abjekt die Einschreibung in Avantgarde-Modelle künstlerischer Rebellion und Außenseitertum bedeutete, bestand für Künstlerinnen eine solche Möglichkeit nur begrenzt (Kort 2003; Ruppert 2018). Gerade die Verknüpfung mit Körper und Sexualität schlug immer wieder in eine affirmative Bestätigung hierarchischer Kreativitätsmodelle um, die Körper mit Weiblichkeit, Männlichkeit dagegen mit intellektueller Bewältigung des Stoffs assoziieren (Zimmermann 2021).

Zweitens bot die Verwendung des Abjektbegriffs zugleich aber auch die Möglichkeit, diese Spaltung kritisch zu analysieren. Die Arbeiten von Eva Hesse waren beispielsweise zunächst sehr stark auf die frühe, zum Tod führende Erkrankung der Künstlerin bezogen worden (Feldhaus 1997). Solche Deutungen duplizierten bestehende Zuweisungen des Abjekten an den weiblichen Körper und bestätigten die Kopplung von Abjekt und Weiblichkeit lediglich. Unter der Perspektive der Abject Art wurde dagegen für eine sorgfältige Trennung zwischen „Werk“ und „Leben“ plädiert (Houser et al. 1993). Die Arbeiten wurden nicht mehr in einem Kurzschluss 
zwischen der „Verworfenheit“ des weiblichen Künstlerinnenkörpers und der ,Verworfenheit" des verwendeten Materials als abjekt gekennzeichnet, sondern konnten in eine viel breitere Deutung integriert werden, die die Kopplung von Weiblichkeit und Abjektion ebenso problematisierte, wie sie die künstlerischen Strategien Hesses nicht nur auf die privaten, körperlichen Erfahrungen der Künstlerin bezog, sondern innerhalb zeitgenössischer künstlerischer Positionen und Entwicklungen situierte.

Drittens schließlich konnte mit dem Konzept der Abject Art der Blick für eine Neubewertung der Arbeiten von Künstlerinnen insgesamt geschärft werden. Vielfach rückten dabei gerade solche Arbeiten in den Blick, die im Umfeld der Neuen Frauenbewegung feministische Themen aufgegriffen hatten (Schor 2015). Sicherlich lässt sich das gestiegene Interesse für diese Werke nicht ausschließlich oder auch nur in erster Linie auf die Prominenz des Abjektbegriffs zurückführen. Aber zumindest thesenhaft sei hier doch formuliert, dass diese Arbeiten, in denen Künstlerinnen Themen wie Körper und Sexualität, Menstruation oder Geburt bearbeiteten, mithilfe des durch Abject Art als kunsthistorischer Kategorie geschulten Blicks in ihrer Bedeutung wahrgenommen werden konnten - jenseits der Fokussierung auf körperlich-privates, wie sie Künstlerinnen lange Zeit zugeschrieben wurde.

\section{Kunst macht das Abjekte als historische Kategorie sichtbar - ein kurzes Fazit}

Was lässt sich aus den hier geschilderten Beispielen über Abject Art, aber durchaus auch über das Abjekt als eine Kategorie, die in unterschiedlichen Disziplinen reflektiert wird und gesellschaftlich wirksam ist, lernen? Abject Art findet zwischen Kunst und Politik statt. Verstanden werden kann sie daher weder aus einer rein ästhetischen Perspektive noch allein über die politischen Reaktionen, die sie auszulösen imstande ist. Setzt man die vorstehenden Beispiele zueinander in Bezug, wird deutlich, wie eng beide Felder zusammenwirken. Bei Andres Serranos schimmerndem Kruzifix sind (politisch wirksamer) Text im Titel und (ästhetisch wirksames) Bild eine unauflösbare Verknüpfung eingegangen. In der Rezeption Eva Hesses und anderer moderner Künstlerinnen, deren Arbeiten als abjekt klassifiziert wurden, führte die Diskussion ästhetischer Aspekte (wie etwa die Auflösung geometrischer Formen als Abgrenzung zum Minimalismus) zur Reflexion politischer Implikationen (wie etwa in Hinblick auf die Rolle von Künstlerinnen). Aus dieser Verbindung ergibt sich noch etwas Drittes. Die Artikulation des Abjekts im künstlerischen Feld und seine unterschiedliche, oft sogar widersprüchliche Rezeption, lässt sich vor diesem Hintergrund als Hinweis auf das Abjekt als historische Kategorie verstehen. Denn die künstlerischen Experimente mit dem Abjekt illustrieren gerade keine überzeitlich gedachten Ekelphänomene, sondern zeigen in Verbindung mit ihrer Rezeptionsgeschichte, inwiefern das, was als abjekt rubriziert wird, sich historisch wandelt und belegen damit auch die unausweichliche politische Aufladung des „Ekelhaften“.

Funding Open Access funding enabled and organized by Projekt DEAL.

Open Access Dieser Artikel wird unter der Creative Commons Namensnennung 4.0 International Lizenz veröffentlicht, welche die Nutzung, Vervielfältigung, Bearbeitung, Verbreitung und Wiedergabe in 
jeglichem Medium und Format erlaubt, sofern Sie den/die ursprünglichen Autor(en) und die Quelle ordnungsgemäß nennen, einen Link zur Creative Commons Lizenz beifügen und angeben, ob Änderungen vorgenommen wurden.

Die in diesem Artikel enthaltenen Bilder und sonstiges Drittmaterial unterliegen ebenfalls der genannten Creative Commons Lizenz, sofern sich aus der Abbildungslegende nichts anderes ergibt. Sofern das betreffende Material nicht unter der genannten Creative Commons Lizenz steht und die betreffende Handlung nicht nach gesetzlichen Vorschriften erlaubt ist, ist für die oben aufgeführten Weiterverwendungen des Materials die Einwilligung des jeweiligen Rechteinhabers einzuholen.

Weitere Details zur Lizenz entnehmen Sie bitte der Lizenzinformation auf http://creativecommons.org/ licenses/by/4.0/deed.de.

\section{Literatur}

Bann, Stephen. 1985. The mythical conception is the name. Titles and names in modern and post-modern painting. Word\&Image 1/2:176-190.

Bruch, Natalie. 2005. Der Bildtitel: Struktur, Bedeutung, Referenz, Wirkung und Funktion, Typologie. Frankfurt a.M.: Peter Lang.

Culler, Jonathan. 1988. Dekonstruktion: Derrida und die poststrukturalistische Literaturtheorie. Reinbek b. Hamburg: Rowohlt.

Cumming, Naomi. 1998. Playing with transgressive light. Serrano's, piss Christ‘. Literature \& Aesthetics $8: 45-59$.

Feldhaus, Reinhild. 1997. Geburt und Tod in Künstlerinnen-Viten der Moderne. Zur Rezeption von Paula Modersohn-Becker, Frida Kahlo und Eva Hesse. In Mythen von Autorschaft und Weiblichkeit im 20. Jahrhundert, Hrsg. Kathrin Hoffmann-Curtius, Silke Wenk, 73-89. Marburg: Jonas.

Hartman, Andrew. 2019. A war for the soul of america: a history of the culture wars. Chicago, London: University of Chicago Press.

Houser, Craig, Leslie C. Jones, Simon Taylor und Jack Ben-Levi (Hrsg.). 1993. Abject art: repulsion and desire in American art. New York: Whitney Museum of American Art.

Korsmeyer, Carolyn. 2011. Savoring disgust: the foul and the fair in aesthetics. Oxford: University Press.

Kort, Pamela (Hrsg.). 2003. Grotesk! 130 Jahre Kunst der Frechheit. München: Prestel.

Kristeva, Julia. 1982. Powers of horror: an essay on abjection. New York: Columbia University Press.

Menninghaus, Winfried. 2002. Ekel. In Ästhetische Grundbegriffe, Bd. 2 (Dekadent - Grotesk), Hrsg. Karlheinz Barck, Martin Pontius, Dieter Schlenstedt, Burkhart Steinwachs, und Friedrich Wolfzettel, 142-177. Stuttgart: J.B. Metzler.

Ruppert, Wolfgang. 2018. Künstler! Kreativität zwischen Mythos, Habitus und Profession. Wien: Böhlau.

Schor, Gabriele (Hrsg.). 2015. Feministische Avantgarde: Kunst der 1970er-Jahre aus der Sammlung Verbund. Wien, München: Prestel.

Silk, Gerald. 1997. Censorship in the career of Edward Kienholz. In Suspended. Censorship in the visual arts, Hrsg. Elisabeth Childs, 259-198. Seattle, London: University of Washington Press.

Vogt, Tobias. 2006. Untitled: Zur Karriere unbetitelter Kunst in der jüngsten Moderne. München: Wilhelm Fink.

Wagner Middleton, Anne. 1994. Three artists (three women): modernism and the art of Hesse, Krasner, and O'Keefe. Berkeley: University of California Press.

Young, Alison. 2000. Aesthetic vertigo and the jurisprudence of disgust. Law and Critique 11:241-265.

Zimmermann, Anja. 2001. Skandalöse Bilder - Skandalöse Körper: Abject Art vom Surrealismus bis zu den Culture Wars. Berlin: Reimer.

Zimmermann, Anja. 2021. Sicherung der Außengrenzen: Transgressive Weiblichkeit, Abject Art und andere Strategien am Rand der Kunst. In Kunst an den Rändern: Wie aus Bildern und Objekten Kunst werden kann, Hrsg. Annika Frye, Christiane Kruse, 162-182. Berlin: De Gruyter. 